\title{
The association between parathyroid hormone 1-34 and the Wnt/ $\beta$-catenin signaling pathway in a rat model of osteoarthritis
}

\author{
LONG MA, JIANG WU and QUN HUA JIN
}

\begin{abstract}
Orthopedics Ward 3, The General Hospital of Ningxia Medical University, Yinchuan, Ningxia 750004, P.R. China
\end{abstract}
Received December 21, 2015; Accepted January 1, 2017

DOI: $10.3892 / \mathrm{mmr} .2017 .7762$

\begin{abstract}
The aim of the current study was to determine the effect of parathyroid hormone (PTH) 1-34 on cartilage degeneration, and the association between PTH 1-34 and factors associated with the $\mathrm{Wnt} / \beta$-catenin pathway following anterior cruciate ligament and medial meniscectomy-induced osteoarthritis (OA) in rats. A total of 64 Sprague-Dawley rats were randomly divided into the following four groups: Sham-operated rats with normal saline (NS)-treatment $(n=16)$; anterior cruciate ligament transection with partial medial meniscectomy (ACLT + MMx) rats with NS-treatment $(n=16)$; sham-operated rats treated with PTH 1-34 $(n=16)$; and ACLT + MMx rats treated with PTH 1-34 $(n=16)$. PTH $(15 \mu \mathrm{g} / \mathrm{kg} /$ day) was administered via subcutaneous injection 5 days per week from the first postoperative day for 2 or 6 weeks. Staining with hematoxylin and eosin and safranin $\mathrm{O}$, and a scoring system modified by Mankin were used to assess the histopathological features of cartilage. The present study detected the expression of PTH 1 receptor (PTH1R), sclerostin, dickkopf Wnt signaling pathway inhibitor 1 (DKK1), $\beta$-catenin and runt-related transcription factor 2 (RUNX2) in cartilage by immunohistochemical analysis to determine the association between PTH 1-34 and factors associated with the Wnt/ $\beta$-catenin pathway. Reverse transcription-quantitative polymerase chain reaction (RT-qPCR) was performed to detect the mRNA expression levels of PTH1R and $\beta$-catenin in cartilage. Histological analysis demonstrated that cartilage degeneration was present post-surgery and gradually increased over time. PTH 1-34 reduced the Mankin scores in ACLT + MMx rats compared with the NS-treated ACLT + MMx rats. Immunohistochemistry and RT-qPCR analysis demonstrated that, in cartilage, PTH 1-34 treatment increased the mRNA
\end{abstract}

Correspondence to: Professor Qun Hua Jin, Orthopedics Ward 3, The General Hospital of Ningxia Medical University, 804 Shengli South Street, Yinchuan, Ningxia 750004, P.R. China

E-mail: jinqunhua@sina.com

Key words: osteoarthritis, cartilage, parathyroid hormone 1-34, Wnt/ $\beta$-catenin signaling pathway, sclerostin, dickkopf Wnt signaling pathway inhibitor 1, runt-related transcription factor 2 expression and protein levels of PTH1R and $\beta$-catenin, and decreased protein levels of sclerostin, DKK1 and RUNX2 in ACLT + MMx rats compared with the NS-treated ACLT + MMx group. The present study demonstrated that PTH 1-34 upregulated the Wnt/ $\beta$-catenin signaling pathway and that PTH1-34 downregulated RUNX2 through an alternative pathway to the $\mathrm{Wnt} / \beta$-catenin signaling pathway, in a rat model of OA.

\section{Introduction}

Osteoarthritis (OA) is one of the most common forms of arthritis. The slow development of the disease affects joint structures, which leads to pain and disability in the elderly. Various risk factors are associated with the initiation and progression of OA, including demographic characteristics and family history, obesity and metabolic syndrome, nutritional factors, bone density and bone mass, and smoking (1). The primary pathological features of OA are cartilage degeneration, marginal osteophyte formation and subchondral bone sclerosis (2). Current treatment strategies are limited to relieving joint pain and improving joint function, and eventually end in artificial joint replacement as no therapeutic strategies that halt the progression of the disease exist (2). Therefore, it is particularly important to clarify the etiology and pathogenesis of OA, which remain unclear. Articular cartilage degeneration is one of the major factors behind the development of OA. Chondrocytes and extracellular matrix are the major components of cartilage, and chondrocyte hypertrophy and extracellular matrix damage may lead to cartilage degeneration $(3,4)$.

Parathyroid hormone (PTH) has roles in the anabolic and catabolic metabolism of G-protein-coupled receptor signaling proteins (5). PTH 1-34 (also termed teriparatide) is the only drug approved by the US Food and Drug Administration for the treatment of osteoporosis (6). Its action is mediated by the parathyroid hormone 1 receptor (PTH1R), which is expressed in chondrocytes (7). Previous studies have demonstrated that PTH 1-34 may prevent OA (8-10). PTH 1-34 inhibits cartilage degeneration and promotes cartilage regeneration following meniscal/ligamentous injury-induced OA in mice (9). PTH 1-34 also prevents the degeneration of articular cartilage and retains the subchondral bone microstructure in spontaneous OA of guinea pigs (8). Furthermore, PTH 1-34 inhibits the terminal differentiation of human articular chondrocytes 
with azacytidine-induced and papain-induced OA in rats (10). However, the specific functions of PTH 1-34 in OA are yet to be established.

$\mathrm{Wnt} / \beta$-catenin signaling pathways have a central role in the maintenance of cartilage homeostasis (3). In cartilage, moderate activity of Wnt is essential for chondrocyte proliferation and the maintenance of typical cartilage characteristics $(4,11,12)$. However, excessive activity of the Wnt/ $\beta$-catenin signaling pathway may result in the hypertrophy of chondrocytes and the degradation of the extracellular matrix of cartilage (11). In cartilage, ablation of $\beta$-catenin increases aggrecan and collagen X (12). Therefore, excessive or inadequate $\beta$-catenin may damage the homeostasis of articular cartilage. PTH and Wnt/ $\beta$-catenin exist in complex associations, which are important for the pathogenesis of OA (3). Sclerostin and dickkopf Wnt signaling pathway inhibitor 1 (DKK1) are upstream inhibitors of the $\mathrm{Wnt} / \beta$-catenin signaling pathway and are used in the research of OA (13). Runt-related transcription factor 2 (RUNX2), which is highly expressed in hypertrophic chondrocytes, regulates the transcription of hypertrophic markers, including collagen $\mathrm{X}$ and matrix metallopeptidase (MMP) 13 (3). The Wnt/ $\beta$-catenin pathway promotes RUNX2 expression (14). In cartilage, the specific association between PTH and RUNX2 is not yet established, and its role in the pathogenesis of OA remains unclear. The present study was designed to investigated the effect of PTH 1-34 on cartilage degeneration and the association between PTH 1-34 and the Wnt/ $\beta$-catenin signaling pathway following anterior cruciate ligament transection (ACLT) and partial medial meniscectomy (MMx) -induced OA in rats.

\section{Materials and methods}

Animal models and treatment. All experiments were approved by the Ningxia Medical University Animal Care and Use Committee (Yinchuan, China). All animal model procedures were carried out according to the principles and guidelines of ethical animal studies. A total of 64 healthy male Sprague-Dawley rats, 10 weeks old and 300-325 g were used in the following experiments. The animals were housed at $22 \pm 2^{\circ} \mathrm{C}$ with $55 \pm 5 \%$ humidity, free access to food and water and 12-h light/dark cycle. Experiments were performed on the right knees of rats in this study. The rats were anesthetized by an intraperitoneal injection of $10 \%$ chloral hydrate (0.3 ml/100 g; Tianjin Guangfu Fine Chemical Research Institute, Tianjin, China). In the OA animal models, the right knee joint cavity was exposed using the medial parapatellar approach. The patella was dislocated laterally when the knee was placed in full flexion. The ACLT in combination with MMx (ACLT + MMx) was performed as previously described (15) and confirmed with the anterior drawer test. (16) Subsequently, the joint surface was washed with sterile saline solution and the knee was closed. In the sham operation, the same procedures were performed with the exception of ACLT + MMx (15). Rats were randomly assigned to four groups: Sham-operated rats with normal saline (NS)-treatment $(n=16)$; ACLT + MMx rats with NS-treatment $(n=16)$; sham-operated rats treated with PTH 1-34 (n=16); and ACLT + MMx rats treated with PTH 1-34 $(n=16)$. PTH 1-34 (Sigma-Aldrich; Merck KGaA, Darmstadt, Germany) was administered by subcutaneous injection
(15 $\mu \mathrm{g} / \mathrm{kg} /$ day) five successive days per week (Monday-Friday) from the first postoperative day until sacrifice at 2 or 6 weeks. Rats not receiving PTH 1-34 received NS at the same dose and duration. Following the sacrifice of rats with carbon dioxide, right knees were divided and the distal femurs were removed for subsequent experiments.

Histological analysis. Femurs were fixed in $4 \%$ paraformaldehyde for $24 \mathrm{~h}$, decalcified in 10\% EDTA for 6 weeks and embedded in paraffin. They were cut into $4 \mu \mathrm{m}$-thick sections and mounted on common slides for staining with hematoxylin and eosin and safranin $\mathrm{O}$. The histopathological features of cartilage were analyzed using the scoring system modified by Mankin et al (17).

Immunohistochemical staining of PTH1R, sclerostin, DKK1, $\beta$-catenin and RUNX2. To investigate the association between PTH 1-34 and factors associated with the Wnt/ $\beta$-catenin pathway, the present study measured the expression of PTH1R, sclerostin, DKK1, $\beta$-catenin and RUNX2 in cartilage by immunohistochemical analysis. Paraffin sections $(4 \mu \mathrm{m})$ of joint tissue were routinely deparaffinized, rehydrated and treated with $0.1 \%$ trypsin for antigen retrieval for $15 \mathrm{~min}$ at $37^{\circ} \mathrm{C}$, and subsequently incubated with $3 \% \mathrm{H}_{2} \mathrm{O}_{2}$ solution for $10 \mathrm{~min}$. Sections were blocked with normal goat serum (Wuhan Boster Biological Technology, Ltd., Wuhan, China) for $8 \mathrm{~min}$ at $37^{\circ} \mathrm{C}$ and incubated overnight at $4^{\circ} \mathrm{C}$ with anti-rabbit sclerostin (1:50; ab63097), DKK1 (1:100; ab93017), $\beta$-catenin (1:100; ab16051) and RUNX2 (1:100; ab23981) polyclonal antibodies (Abcam, Cambridge, UK) and anti-rabbit PTH1R polyclonal antibody (1:100; BA3170; Wuhan Boster Biological Technology, Ltd.). Sections were then treated with the Two-Step IHC Detection Reagent (PV-9001; Origene Technologies, Inc., Rockville, MD, USA). Briefly, sections were incubated with reagent 1 (Reaction enhancement solution) for $20 \mathrm{~min}$ at $37^{\circ} \mathrm{C}$ and then incubated with reagent 2 (Enhanced enzyme-labeled goat anti-rabbit IgG polymer) for $30 \mathrm{~min}$ at $37^{\circ} \mathrm{C}$. The color brown was developed using 3,3'-diaminobenzidine (Origene Technologies, Inc.). For negative controls, PBS without antibody was used for incubation. Counterstaining was carried out with hematoxylin. The positive cells were stained yellow or brown. All sections were analyzed using Image-Pro Plus 6.0 (Media Cybernetics, Inc., Rockville, MD, USA) at x400 magnification to quantify protein expression; 5 random fields were randomly selected in each slide and the numbers of positive cells were counted and expressed as a percentage of the total cells.

Reverse transcription-quantitative polymerase chain reaction $(R T-q P C R)$. Femurs were thoroughly cleaned and the muscle, ligament and joint capsule were simultaneously removed, while damage to the femur was avoided. The femur was rapidly frozen with liquid nitrogen and articular cartilage was obtained for further investigation. Following treatment, total RNA was extracted with an E.Z.N.A Total RNA kit (Omega Bio-Tek, Inc., Norcross, GA, USA) from articular cartilage according to the manufacturer's protocol. Subsequently, total RNA was reverse transcribed to cDNA using RevertAid First Strand cDNA Synthesis kit (Thermo Fisher Scientific, Inc., Waltham, MA, USA) according to the manufacturer's protocol. Specific transcripts were quantified by qPCR using Maxima 

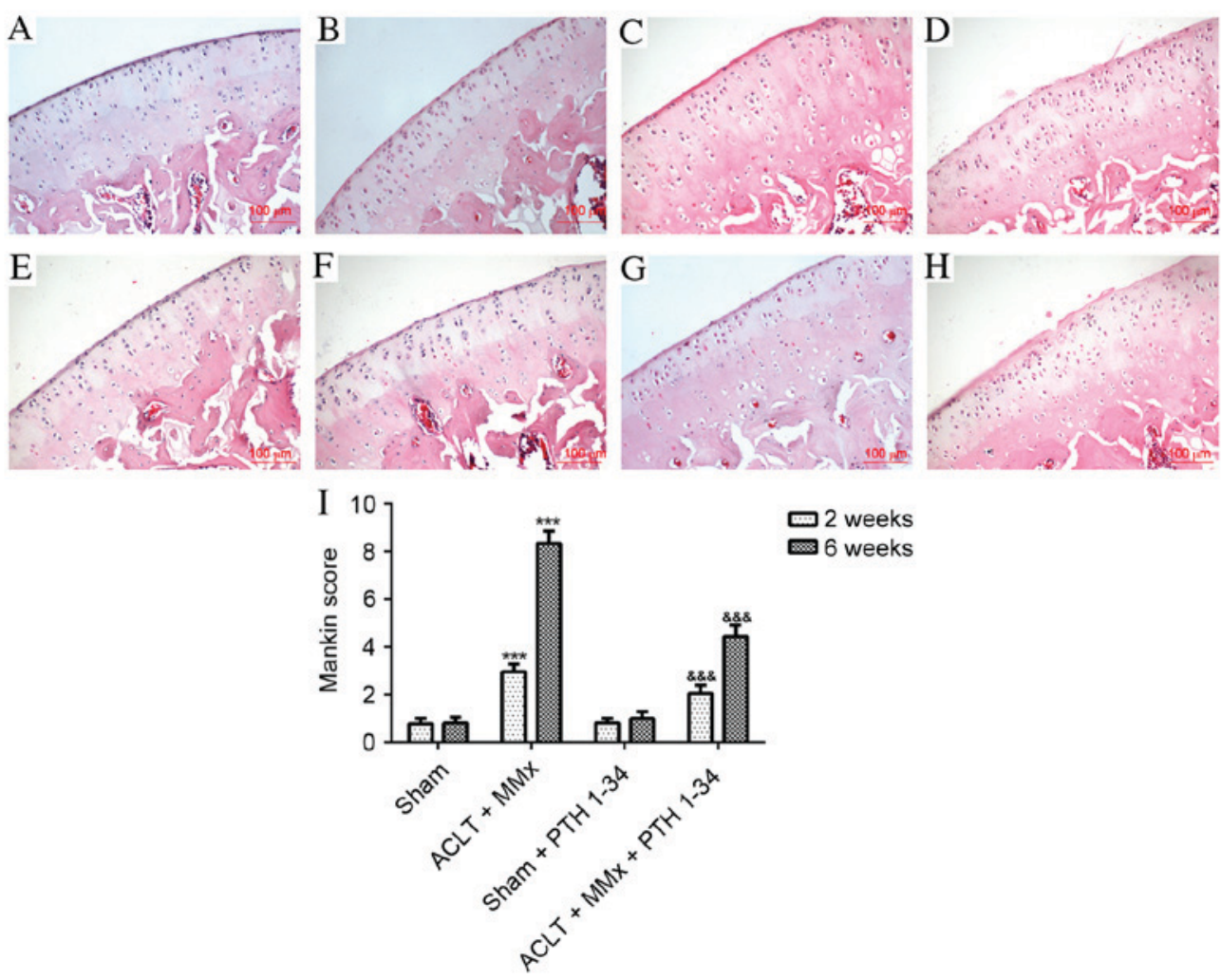

四 2 weeks

6 weeks

Figure 1. Histological analysis of the articular cartilage of the distal femur by hematoxylin and eosin staining, and Mankin score. Sham-operated rats with NS treatment after (A) 2 weeks and (B) 6 weeks. ACLT + MMx rats with NS treatment after (C) 2 weeks and (D) 6 weeks. Sham-operated rats with PTH 1-34 treatment after (E) 2 weeks and (F) 6 weeks. ACLT + MMx rats with PTH 1-34 treatment after (G) 2 weeks and (H) 6 weeks. x200 magnification. (I) Mankin scores from each group ( 5 slides/rat and 8 rats/group). ${ }^{* * *} \mathrm{P}<0.001 \mathrm{vs.} \mathrm{Sham} \mathrm{group;}{ }^{\& \&} \mathrm{P}<0.001$ vs. ACLT $+\mathrm{MMx}$ group. ACLT, anterior cruciate ligament transection; MMx, partial medial meniscectomy; PTH, parathyroid hormone; NS, normal saline.

SYBR-Green qPCR Kit (Thermo Fisher Scientific, Inc.), and analyzed with an ABI 7500 Real-Time PCR system (Applied Biosystems; Thermo Fisher Scientific, Inc.). The following gene-specific primers were used: PTH1R, TCTCCTTAC CCAGGCAGATG (forward) and CATTGCATCCTCTCC ACAGA (reverse); $\beta$-catenin, ACCATCGAGAGGGCTTGT TG (forward) and CGCACTGCCATTTTAGCTCC (reverse); and GAPDH, GAAGGTGAAGGTCGGAGTC (forward) and GAAGATGGTGATGGGATTTC (reverse). PCR was performed at $95^{\circ} \mathrm{C}$ for $10 \mathrm{~min}$, followed by 40 cycles of $95^{\circ} \mathrm{C}$ for $15 \mathrm{sec}$, and $60^{\circ} \mathrm{C}$ for $1 \mathrm{~min}$. Expression of PTH1R and $\beta$-catenin mRNA was calculated using the $\Delta \Delta$ Cq method (18), levels were normalized to the expression of GAPDH. Each experiment was repeated three times with each rat.

Statistical analysis. SPSS version 20.0 (SPSS, Inc., Chicago, IL, USA) was used for statistical analysis. Results were analyzed using two-way factorial design analysis of variation followed by Student-Newman-Keuls post-hoc test. Data are presented as the mean \pm standard deviation. $\mathrm{P}<0.05$ was considered to indicate a statistically significant difference.

\section{Results}

Histological assessments. The present study applied hematoxylin and eosin staining (Fig. 1), safranin O staining (Fig. 2) and the Mankin score (Figs. 1 and 2) to evaluate the effect of PTH 1-34 on the knee joints of ACLT + MMx rats. The articular cartilage in NS-treated sham rats and PTH 1-34-treated sham rats at 2 and 6 weeks had a normal, smooth surface and structure, and no abnormalities were observed. Early histopathological changes were observed after 2 weeks in NS-treated ACLT + MMx rats, including chondrocytes with diffuse hyper-cellularity, a marginal reduction in matrix staining and destruction of tidemark integrity. After 6 weeks in NS-treated ACLT + MMx rats, more serious histological changes were observed, including structure clefts to transitional zone, chondrocyte clusters, a severe reduction in matrix staining and destruction of tidemark integrity. Histological scoring was performed using the modified Mankin histological scores to evaluate pathological changes of articular cartilage. After 2 and 6 weeks, ACLT + MMx rats receiving PTH 1-34 treatment had reduced articular cartilage degeneration, compared with the NS-treated ACLT + MMx rats. The present study also observed that there were no marked differences between the PTH 1-34-treated sham rats and NS-treated sham rats. For the ACLT + MMx rats, the Mankin score increased with time compared with the sham rats. The Mankin score of the hematoxylin and eosin (Fig. 1) and safranin O (Fig. 2) staining in PTH 1-34-treated ACLT + MMx rats, decreased by 31 and $46 \%$, and 36 and $51 \%$, at 2 and 6 weeks, respectively, compared with NS-treated ACLT + MMx rats.

Immunohistochemical analysis. The expression of PTH1R, sclerostin, DKK1, $\beta$-catenin and RUNX2 in cartilage was evaluated by immunohistochemistry assays. The expression 

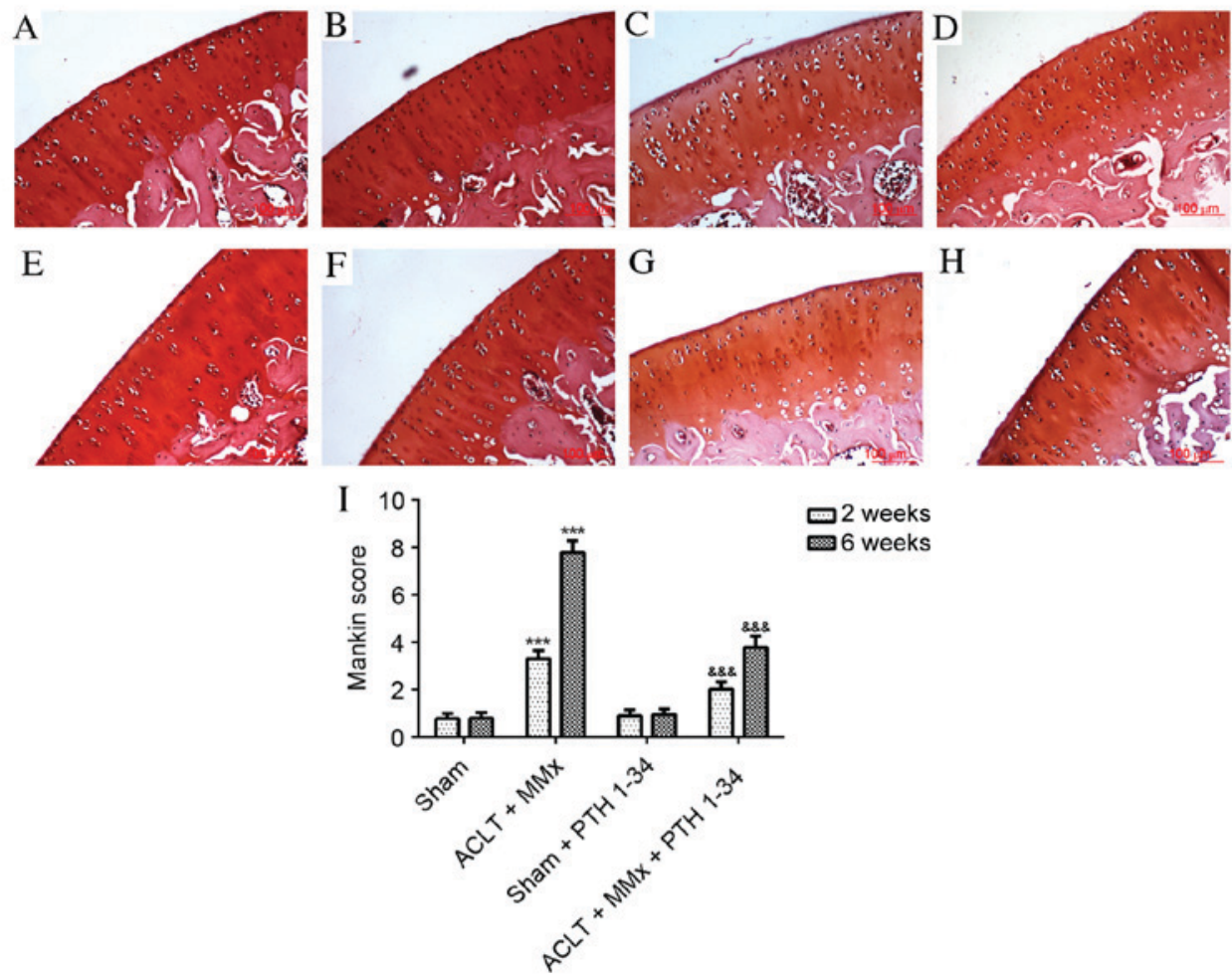

四 2 weeks

6 weeks

Figure 2. Histological results in articular cartilage of distal femur by safranin O staining and Mankin score. Sham-operated rats with NS-treatment after (A) 2 weeks and (B) 6 weeks. ACLT + MMx rats with NS-treatment after (C) 2 weeks and (D) 6 weeks. Sham-operated rats with PTH 1-34-treatment after (E) 2 weeks and (F) 6 weeks. ACLT + MMx rats with PTH 1-34-treatment after (G) 2 weeks and (H) 6 weeks. x200 magnification. (I) Mankin scores from each group (5 slides/rat and 8 rats/group). ${ }^{* * *} \mathrm{P}<0.001$ vs. Sham group; ${ }^{\& \&} \& \mathrm{P}<0.001$ vs. ACLT + MMx group. ACLT, anterior cruciate ligament transection; MMx, partial medial meniscectomy; PTH, parathyroid hormone; NS, normal saline.
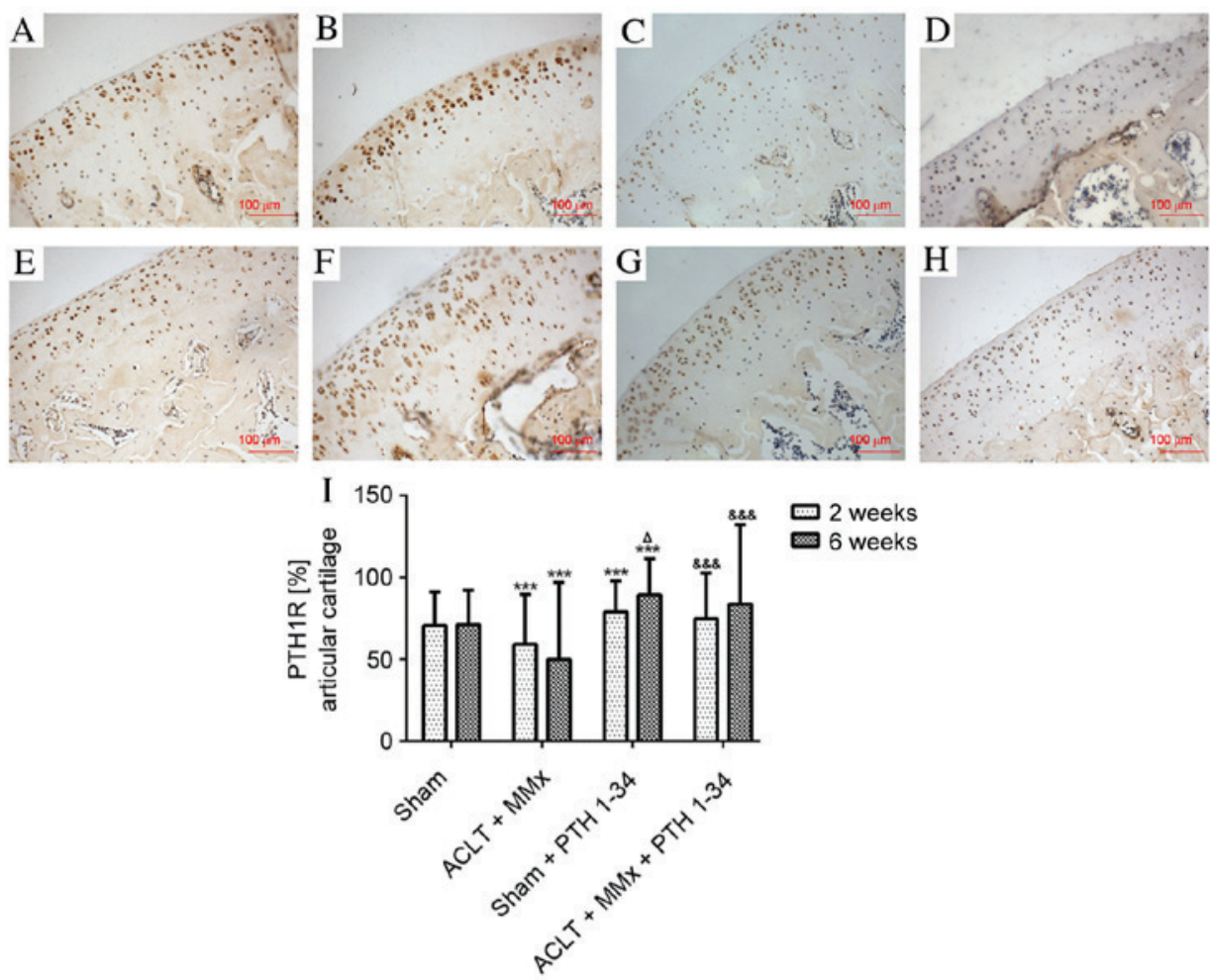

Figure 3. Immunohistochemistry analysis of PTH1R expression in cartilage. Sham-operated rats with NS-treatment after (A) 2 weeks and (B) 6 weeks. ACLT + MMx rats with NS-treatment after (C) 2 weeks and (D) 6 weeks. Sham-operated rats with PTH 1-34-treatment after (E) 2 weeks and (F) 6 weeks. ACLT + MMx rats with PTH 1-34-treatment after (G) 2 weeks and (H) 6 weeks. x200 magnification. (I) Protein expression of PTH1R in each group. ${ }^{* * *} \mathrm{P}<0.001$ vs. Sham group; ${ }^{\& \& \&} \mathrm{P}<0.001$ vs. ACLT $+\mathrm{MMx}$ group; ${ }^{\wedge} \mathrm{P}<0.05$ vs. PTH 1-34-treated sham group at 2 weeks. PTH, parathyroid hormone; PTH1R, PTH 1 receptor; ACLT, anterior cruciate ligament transection; MMx, partial medial meniscectomy; NS, normal saline. 

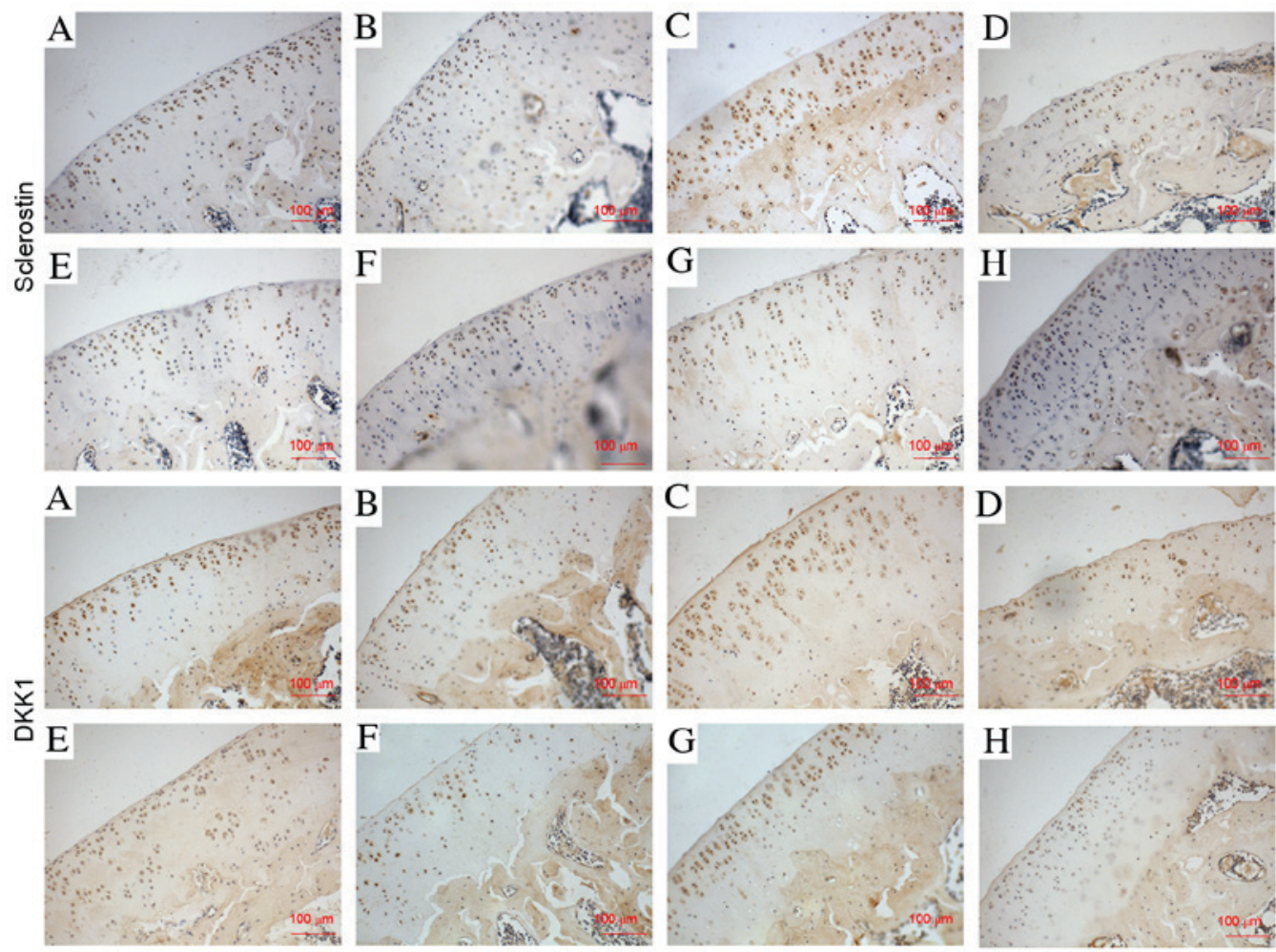

Figure 4. Immunohistochemistry assay for sclerostin and DKK1 in cartilage. Sham-operated rats with NS-treatment after (A) 2 weeks and (B) 6 weeks ACLT + MMx rats with NS-treatment after (C) 2 weeks and (D) 6 weeks. Sham-operated rats with PTH 1-34-treatment after (E) 2 weeks and (F) 6 weeks. ACLT + MMx rats with PTH 1-34-treatment after (G) 2 weeks and (H) 6 weeks. x200 magnification. DKK1, dickkopf WNT signaling pathway inhibitor 1; NS, normal saline; ACLT, anterior cruciate ligament transection; MMx, partial medial meniscectomy; PTH, parathyroid hormone.

of PTH1R was significantly decreased $(\mathrm{P}<0.001$; Fig. 3), while sclerostin and DKK1 (Figs. 4 and 5), and $\beta$-catenin and RUNX2 (Figs. 6 and 7) were significantly increased in the NS-treated ACLT + MMx rats compared with the NS-treated sham rats at 2 and 6 weeks $(\mathrm{P}<0.001)$. Compared with NS-treated sham rats, the expression of PTH1R was increased by 12 and $25 \%(\mathrm{P}<0.001$; Fig. 3$)$, and $\beta$-catenin was increased by 76 and $81 \%(\mathrm{P}<0.001$; Fig. 7$)$ at 2 and 6 weeks, respectively, in the PTH 1-34-treated sham rats. Furthermore, the expression of sclerostin was decreased by 16 and $27 \%$ ( $\mathrm{P}<0.01$; Fig. 5$)$, DKK1 was decreased by 10 and $23 \%(\mathrm{P}<0.05$; Fig. 5), and RUNX2 was decreased by 10 and $42 \%(\mathrm{P}<0.01$; Fig. 7$)$ at 2 and 6 weeks, respectively, in PTH 1-34-treated sham rats compared with NS-treated sham rats. The expression of PTH1R was increased by 27 and $67 \%(\mathrm{P}<0.001 ;$ Fig. 3$)$, and $\beta$-catenin was increased by 6 and $8 \%(P<0.05$; Fig. 7), while the expression of sclerostin was decreased by 16 and $54 \%(\mathrm{P}<0.001$; Fig. 5), DKK1 was decreased by 10 and 28\% ( $\mathrm{P}<0.001$; Fig. 5), and RUNX2 was decreased by 9 and $19 \%(\mathrm{P}<0.01$; Fig. 7$)$ at 2 and 6 weeks, respectively, in the PTH 1-34-treated ACLT + MMx rats compared with the NS-treated ACLT + MMx rats.

$R T-q P C R$ analysis. To further investigate the mechanism, RT-qPCR was performed to detect the mRNA expression levels of PTH1R and $\beta$-catenin in cartilage (Fig. 8). At 2 and 6 weeks, the gene expression of PTH1R (Fig. 8) was decreased by 15 and $25 \%$, respectively, in NS-treated ACLT + MMx rats compared with NS-treated sham rats $(\mathrm{P}<0.001)$. PTH1R expression was increased by 20 and $40 \%$ in PTH 1-34-treated
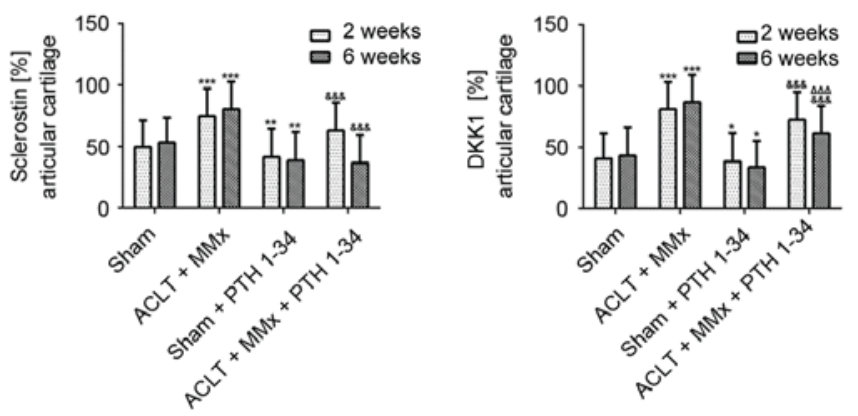

Figure 5. Immunohistochemistry quantification of sclerostin and DKK1 in cartilage (5 slides/rat and 8 rats/group). Sclerostin and DKK1 \% indicate the percentage of positive cells in total cell count. ${ }^{*} \mathrm{P}<0.05,{ }^{* *} \mathrm{P}<0.01$ and ${ }^{* * *} \mathrm{P}<0.001$ vs. Sham group; ${ }^{\& \& \&} \mathrm{P}<0.001$ vs. ACLT + MMx group; ${ }^{\Delta \Delta \Delta} \mathrm{P}<0.001$ vs. ACLT + MMx group at 2 weeks. ACLT, anterior cruciate ligament transection; MMx, partial medial meniscectomy; PTH, parathyroid hormone; DKK1, dickkopf WNT signaling pathway inhibitor 1.

sham rats compared with NS-treated sham rats $(\mathrm{P}<0.001)$, and was increased by 24 and $51 \%$ in PTH 1-34-treated ACLT + MMx rats compared with NS-treated ACLT + MMx rats $(\mathrm{P}<0.001)$. The present study also demonstrated that the gene expression of $\beta$-catenin (Fig. 8) was increased by 150 and $185 \%$ in NS-treated ACLT + MMx rats compared with NS-treated sham rats $(\mathrm{P}<0.001)$, was increased by 101 and $116 \%$ in PTH 1-34-treated sham rats compared with NS-treated sham rats $(\mathrm{P}<0.001)$, and was increased by 8 and 9\% in PTH 1-34-treated ACLT + MMx rats compared with NS-treated ACLT + MMx rats $(\mathrm{P}<0.05)$. 

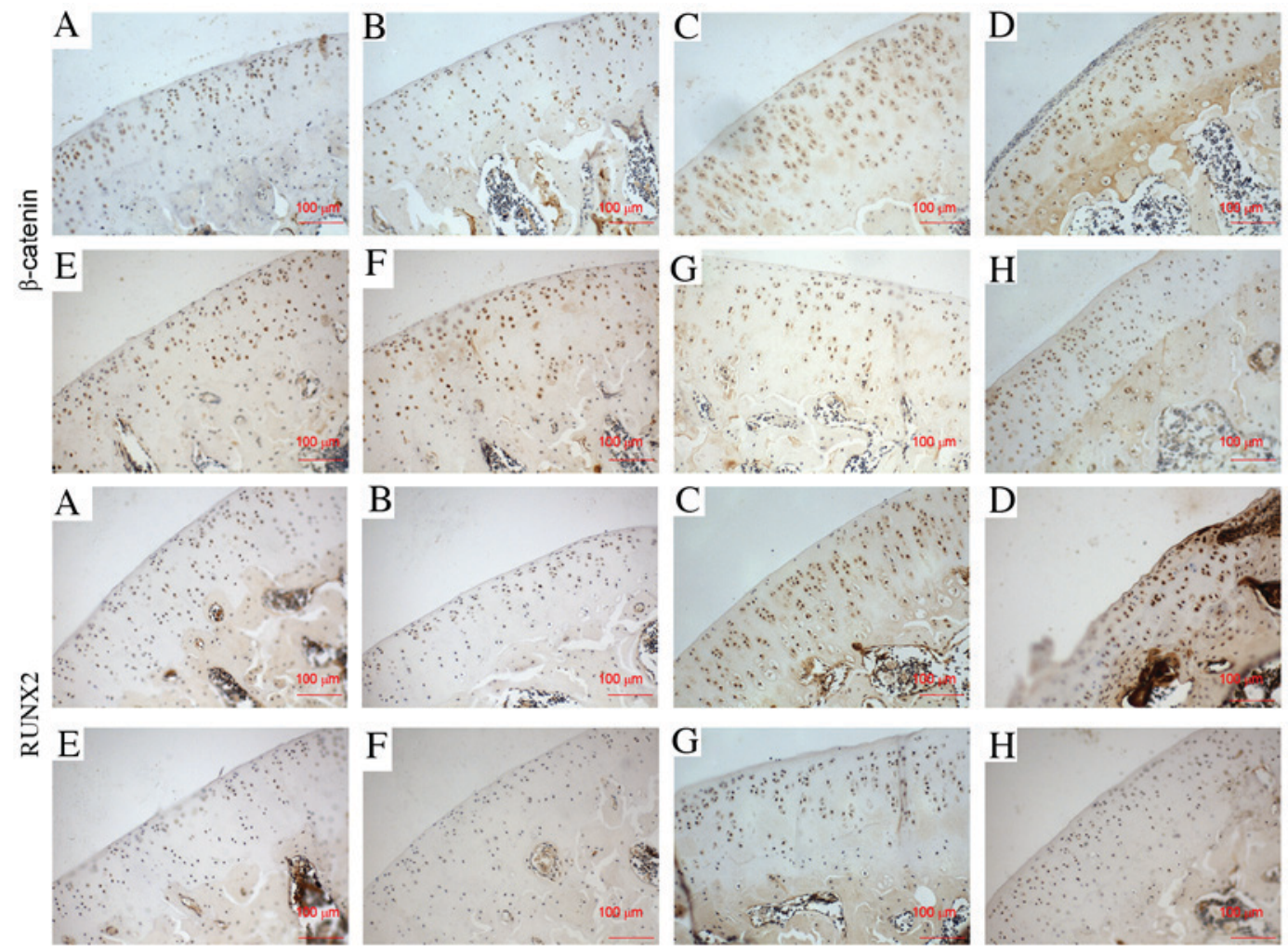

Figure 6. Immunohistochemistry assay for $\beta$-catenin and RUNX2 in cartilage ( 5 slides/rat and 8 rats/group). $\beta$-catenin and RUNX2 \% indicated the percentage of positive cells in total cell count. Sham-operated rats with NS-treatment after (A) 2 weeks and (B) 6 weeks. ACLT + MMx rats with NS-treatment after (C) 2 weeks and (D) 6 weeks. Sham-operated rats with PTH 1-34-treatment after (E) 2 weeks and (F) 6 weeks. ACLT + MMx rats with PTH 1-34-treatment after (G) 2 weeks and (H) 6 weeks. x200 magnification. RUNX2, runt-related transcription factor 2; NS, normal saline; ACLT, anterior cruciate ligament transection; MMx, partial medial meniscectomy; PTH, parathyroid hormone.
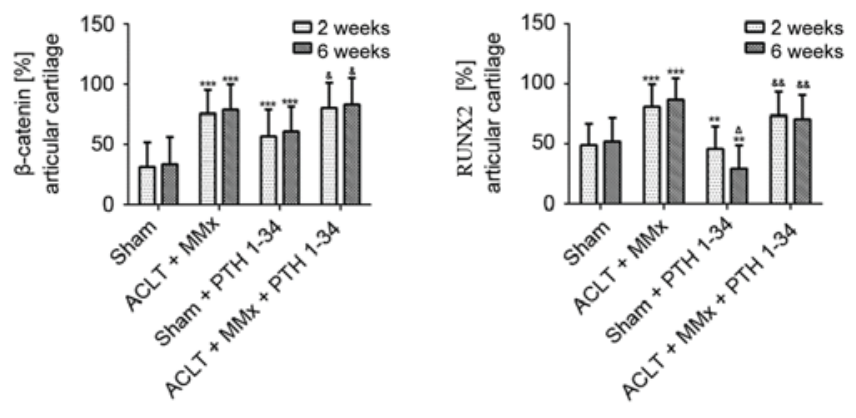

Figure 7. Quantified protein levels of $\beta$-catenin and RUNX2 in cartilage. ${ }^{* *} \mathrm{P}<0.01$ and ${ }^{* * * *} \mathrm{P}<0.001$ vs. Sham group; ${ }^{\&} \mathrm{P}<0.05$ and ${ }^{\& \&} \mathrm{P}<0.01$ vs. ACLT + MMx group; ${ }^{\wedge} \mathrm{P}<0.05$ vs. PTH 1-34-treated sham group at 2 weeks. RUNX2, runt-related transcription factor 2; ACLT, anterior cruciate ligament transection; MMx, partial medial meniscectomy; $\mathrm{PTH}$, parathyroid hormone.

\section{Discussion}

OA is a common degenerative disease that seriously affects daily life and causes an economic burden. The pathogenesis of OA is complex. Cartilage matrix degeneration and hypertrophic chondrocytes have key roles in the progression of OA, therefore, it is particularly important to inhibit the development of these factors. The present study demonstrated that PTH 1-34 reduced the Mankin scores and increased the mRNA expression and protein levels of PTH1R and $\beta$-catenin, and decreased protein levels of sclerostin, DKK1 and RUNX2, in ACLT + MMx rats with PTH 1-34-treatment compared with ACLT + MMx rats with NS-treatment. Similar results for mRNA expression and protein levels were observed between sham rats with PTH 1-34-treatment and sham rats with NS-treatment. These results have, to the best of our knowledge, demonstrated for the first time that PTH 1-34 may upregulate the Wnt/ $\beta$-catenin signaling pathway, and that PTH 1-34 may downregulate RUNX2 through an alternative pathway to the $\mathrm{Wnt} / \beta$-catenin signaling pathway in a rat model of OA.

In OA experimental animal models, surgically-induced destabilization of joints is the most widely used induction method. These models control the timing and follow predictable progression of the disease. Of these OA models, the ACLT + MMx rat model is one of the most common (19). Early cartilage degradation, subchondral osteopenia followed by sclerosis and late osteophyte formation emerge sequentially in the ACLT + MMx rat model (15). In the present study, the ACLT + MMx rat model exhibited cartilage degeneration at 2 weeks post-surgery, which gradually increased over time.

In guinea pigs with spontaneous OA, papain-induced OA in rats, meniscal/ligamentous injury-induced $\mathrm{OA}$ in mice or in osteochondral defects in rabbits, PTH1R expression gradually decreased with progression of OA. Systemic application of PTH 1-34 significantly upregulated PTH1R expression in cartilage $(8-10,20)$. Similar to these previous results, the present study demonstrated that, as OA progressed, the protein levels and mRNA expression of PTH1R gradually declined and systemic application of PTH 1-34 significantly upregulated them in cartilage in the ACLT + MMx rat model. 

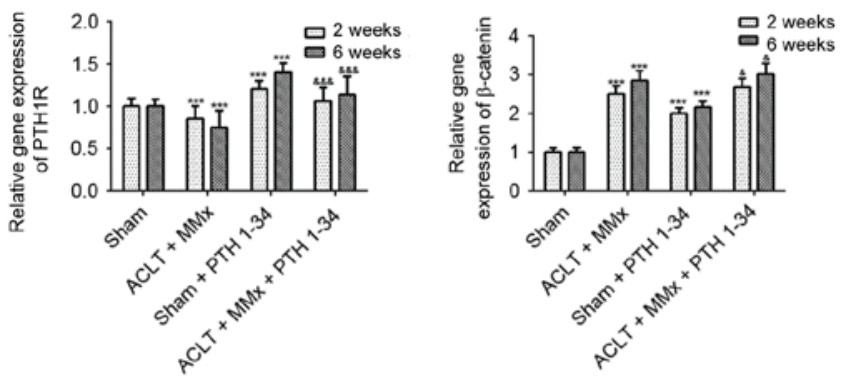

Figure 8 . Reverse transcription-quantitative polymerase chain reaction analyses of PTHR1 and $\beta$-catenin gene expression in cartilage. ${ }^{* * *} \mathrm{P}<0.001$ vs. Sham group; ${ }^{\&} \mathrm{P}<0.05$ and ${ }^{\& \& \&} \mathrm{P}<0.001$ vs. ACLT $+\mathrm{MMx}$ group. $\mathrm{PTH}$, parathyroid hormone; PTH1R, PTH 1 receptor; ACLT, anterior cruciate ligament transection; MMx, partial medial meniscectomy.

Sclerostin, a potent inhibitor of $\mathrm{Wnt} / \beta$-catenin signaling that binds to LDL receptor related protein (LRP)5/6, is encoded by the SOST gene and is expressed by osteocytes and articular chondrocytes. Sclerostin is increased in OA cartilage compared with normal cartilage $(8,21-23)$. In bone, inhibiting sclerostin increases bone formation, bone mass and bone strength (24). The role of sclerostin in OA progression is not well established. Knockout of the SOST gene promoted OA in mice via $\beta$-catenin-dependent and -independent Wnt pathways, therefore, sclerostin has a role in the maintenance of cartilage integrity in OA (25). However, increasing sclerostin using a recombinant sclerostin may retard cartilage degradation (26). The present study demonstrated that with the progression of OA, sclerostin expression gradually increases. To the best of our knowledge, the present study is the first to demonstrate that administration of PTH 1-34 reduced sclerostin expression in cartilage in the ACLT + MMx rat model, similar to guinea pigs with spontaneous OA (8).

DKK1 is a secreted protein and a member of a multigene family. It acts as a direct inhibitor of $\mathrm{Wnt} / \beta$-catenin signaling by binding to LRP5/6 and forms a ternary complex with Kremen and LRP6. DKK1 is a master regulator of joint remodeling (27,28). Various studies have indicated that DKK1 is associated with OA development, however, these results are controversial. A previous study demonstrated that, as joint severity was increased, DKK1 levels were decreased in plasma and synovial fluid, thus, DKK1 may have a role in the progression of OA (29). Previous results demonstrated that DKK1 was increased in OA cartilage when compared with normal cartilage (30). In a previous study that used the destabilization of the medial meniscus mouse model of OA, which was performed using intra-articular injection of an adenovirus expression DKK1 to increase DKK1 expression in chondrocytes, DKK1 inhibited cartilage destruction (30). Increased DKK1 expression in ACLT and collagenase-induced OA rat models, via intra-peritoneal injection of DKK1 antisense oligonucleotides, was previously observed to downregulate DKK1 expression and subsequently ameliorate chondrocyte apoptosis and cartilage destruction in OA (31). The present study demonstrated that DKK1 expression gradually increased with OA progression, and that administration of PTH 1-34 downregulated DKK1 expression in cartilage in the ACLT + MMx rat model. A previous report demonstrated that PTH 1-34 inhibited the expression of DKK1 in osteoblasts (32). Although the results of the present study also demonstrated a similar inhibition of PTH 1-34 on DKK1, the results of the present study were in OA cartilage, and further investigation is required to determine the specific mechanisms involved.

Signaling pathways that control joint and articular cartilage are particularly important for the treatment of OA. The hypertrophy of chondrocytes and degradation of the extracellular matrix of cartilage lead to a loss of articular cartilage that is characteristic of OA (3). Wnt signaling has major roles in the majority of aspects of skeletal development and homeostasis, and abnormal signaling causes various human skeletal diseases (11). The Wnt pathway is a key regulator of joint remodeling and also has a critical role in OA pathogenesis. Wnt/ $\beta$-catenin signaling is associated with a loss of differentiated phenotype and chondrocyte matrix catabolic action, which may contribute to cartilage destruction (33). $\beta$-catenin is a key factor of the Wnt/ $\beta$-catenin pathway that is encoded by the CTNNB1 gene. The nuclear levels of $\beta$-catenin directly reflect the activation level of this signaling pathway. $\beta$-catenin is stable in the cytoplasm. Upon entering the nucleus, it interacts with a transcription factor and subsequently forms a complex with TCF/LEF transcription factors and regulates the expression of target genes (34). Conditional activation of the $\beta$-catenin gene in articular chondrocytes increases MMP-9, MMP-13, alkaline phosphatase and collagen $\mathrm{X}$ in chondrocytes, which leads to premature chondrocyte differentiation and the development of an OA-like phenotype in mice (35). The results of the present study demonstrated that the protein levels and mRNA expression of $\beta$-catenin gradually increased with OA progression, and administration of PTH 1-34 upregulated the protein levels and mRNA expression of $\beta$-catenin in cartilage in the ACLT + MMx rat model. According to a study in osteoblasts, PTH activated $\beta$-catenin via recruitment of LRP6 to the PTH/PTH1R complex (36). PTH increased $\beta$-catenin expression by protein kinase A (PKA) and PKC pathways, and SMAD3 in mouse osteoblastic cells (37). A previous study also demonstrated that PTH1R activated $\beta$-catenin via direct recruitment of disheveled segment polarity proteins, independent of Wnt or LRP5/6, which induced osteoclastogenesis (38). These studies demonstrated that PTH increases the expression of $\beta$-catenin in osteoblasts and osteoclasts. Sclerostin and DKKs negatively regulate Wnt/ $\beta$-catenin signaling in bone formation, and PTH inhibits the expression of sclerostin and DKK1. The current study demonstrated that PTH 1-34 upregulated $\beta$-catenin in cartilage in the $\mathrm{ACLT}+\mathrm{MMx}$ rat model. PTH may increase $\beta$-catenin expression by decreasing sclerostin and DKK1 expression. However, identification of the specific mechanism requires further investigation.

RUNX2, a transcription factor that belongs to the runt homology domain protein family, is strongly expressed in osteoblasts, and also in pre-hypertrophic and hypertrophic chondrocytes $(39,40)$. RUNX2 is a major positive regulator of chondrocyte differentiation (39). RUNX2 is constitutively expressed in non-hypertrophic chondrocytes that induce hypertrophic chondrocyte differentiation and partially rescued the chondrocyte phenotype in RUNX2-deficient mice (41). In mice, following induction of joint instability, RUNX2 may successively induce type X collagen and MMP-13 expression, 
which contribute to chondrocyte hypertrophy and matrix breakdown, therefore causing the pathogenesis of OA (41). Inhibition of RUNX2 delayed endochondral ossification or chondrocyte hypertrophy in a previous study (42). The present study demonstrated that RUNX2 expression gradually increased with OA progression and administration of PTH 1-34 downregulated RUNX2 expression in cartilage in the ACLT + MMx rat model. The association between increased expression of RUNX2 in articular cartilage and articular cartilage degeneration was similar to a previous report (41). A previous study demonstrated that $\mathrm{Wnt} / \beta$-catenin signaling induced chondrocyte hypertrophy through RUNX2 (41). The current study demonstrated that the expression of $\beta$-catenin and RUNX2 was increased in the cartilage of ACLT + MMx rats with NS-treatment compared with NS-treated sham rats. Furthermore, in the PTH 1-34-treated ACLT + MMx rats, the expression of $\beta$-catenin was increased, and the expression of RUNX2 decreased. Increasing $\beta$-catenin did not upregulate RUNX2, which contradicts the results of a previous study (14). Parathyroid hormone like hormone (PTHrP) and PTH have similar N-terminal regions, activate the same G-protein-coupled receptor, PTH1R, and also have similar potential effects (7). A previous study reported that PTHrP downregulated RUNX2 expression, which was accompanied by suppression of type $\mathrm{X}$ collagen through the PKA signaling pathway (43). Therefore, PTH 1-34 may decrease RUNX2 levels through pathways other than the Wnt/ $\beta$-catenin signaling pathway.

In conclusion, the results of the present study indicate that intermittent application of the anabolic bone agent, PTH 1-34, reduced the Mankin scores in the OA model, ACLT + MMx, compared with NS-treated ACLT + MMx rats, with no significant effect observed in sham rats. This may be associated with PTH 1-34 increasing the expression of PTH1R and $\beta$-catenin, and reducing the expression of sclerostin, DKK1 and RUNX2 in cartilage. Although the experimental models used in the present study allowed us to control the time of disease onset and follow predictable progression of the disease, rats have a faster growth rate and higher disease severity than humans and they do not completely imitate OA development in humans. The present study investigated the association between PTH1R and the factors associated with the Wnt/ $\beta$-catenin pathway, and did not detect downstream factors, including type X collagen and MMP-13. Therefore, further investigation is needed to determine the association between PTH 1-34 and downstream factors in articular cartilage. Additionally, integration of information from different signaling pathways is required, which may provide useful information for the investigation of the pathogenesis of OA.

\section{References}

1. Allen KD and Golightly YM: State of the evidence. Curr Opin Rheumatol 27: 276-283, 2015.

2. Pulsatelli L, Addimanda O, Brusi V, Pavloska B and Meliconi R: New findings in osteoarthritis pathogenesis: Therapeutic implications. Ther Adv Chronic Dis 4: 23-43, 2013.

3. Zhong L, Huang X, Karperien M and Post JN: The regulatory role of signaling crosstalk in hypertrophy of MSCs and human articular chondrocytes. Int J Mol Sci 16: 19225-19247, 2015.

4. Yuasa T, Otani T, Koike T, Iwamoto M and Enomoto-Iwamoto M: Wnt/beta-catenin signaling stimulates matrix catabolic genes and activity in articular chondrocytes: Its possible role in joint degeneration. Lab Invest 88: 264-274, 2008.
5. Harrington EK, Roddy GW, West R and Svoboda KK: Parathyroid hormone/parathyroid hormone-related peptide modulates growth of avian sternal cartilage via chondrocytic proliferation. Anat Rec (Hoboken) 290: 155-167, 2007.

6. Neer RM, Arnaud CD, Zanchetta JR, Prince R, Gaich GA, Reginster JY, Hodsman AB, Eriksen EF, Ish-Shalom S, Genant HK, et al: Effect of parathyroid hormone (1-34) on fractures and bone mineral density in postmenopausal women with osteoporosis. N Engl J Med 344: 1434-1441, 2001.

7. Abou-Samra AB, Jüppner H, Force T, Freeman MW, Kong XF, Schipani E, Urena P, Richards J, Bonventre JV, Potts JT Jr, et al: Expression cloning of a common receptor for parathyroid hormone and parathyroid hormone-related peptide from rat osteoblast-like cells: A single receptor stimulates intracellular accumulation of both cAMP and inositol trisphosphates and increases intracellular free calcium. Proc Natl Acad Sci USA 89: 2732-2736, 1992.

8. Yan JY, Tian FM, Wang WY, Cheng Y, Song HP, Zhang YZ and Zhang L: Parathyroid hormone (1-34) prevents cartilage degradation and preserves subchondral bone micro-architecture in guinea pigs with spontaneous osteoarthritis. Osteoarthritis Cartilage 22: 1869-1877, 2014.

9. Sampson ER, Hilton MJ, Tian Y, Chen D, Schwarz EM, Mooney RA, Bukata SV, O'Keefe RJ, Awad H, Puzas JE, et al: Teriparatide as a chondroregenerative therapy for injury-induced osteoarthritis. Sci Transl Med 3: 101ra93, 2011.

10. Chang JK, Chang LH, Hung SH, Wu SC, Lee HY, Lin YS, Chen $\mathrm{CH}, \mathrm{Fu}$ YC, Wang GJ and Ho ML: Parathyroid hormone 1-34 inhibits terminal differentiation of human articular chondrocytes and osteoarthritis progression in rats. Arthritis Rheum 60: 3049-3060, 2009.

11. Regard JB, Zhong Z, Williams BO and Yang Y: Wnt signaling in bone development and disease: Making stronger bone with Wnts. Cold Spring Harb Perspect Biol 4: pii: a007997, 2012.

12. Yasuhara R, Ohta Y, Yuasa T, Kondo N, Hoang T, Addya S, Fortina $\mathrm{P}$, Pacifici M, Iwamoto $\mathrm{M}$ and Enomoto-Iwamoto $\mathrm{M}$ : Roles of $\beta$-catenin signaling in phenotypic expression and proliferation of articular cartilage superficial zone cells. Lab Invest 91: 1739-1752, 2011.

13. Loeser RF: Osteoarthritis Year in Review 2013: Biology. Osteoarthritis Cartilage 21: 1436-1442, 2013.

14. Dong YF, Soung do Y, Schwarz EM, O'Keefe RJ and Drissi H: Wnt induction of chondrocyte hypertrophy through the Runx2 transcription factor. J Cell Physiol 208: 77-86, 2006.

15. Pickarski M, Hayami T,Zhuo Y and Duong LT: Molecular changes in articular cartilage and subchondral bone in the rat anterior cruciate ligament transection and meniscectomized models of osteoarthritis. BMC Musculoskelet Disord 12: 197, 2011.

16. Rossi R, Dettoni F, Bruzzone M, Cottino U, D'Elicio DG and Bonasia DE: Clinical examination of the knee: Know your tools for diagnosis of knee injuries. Sports Med Arthrosc Rehabil Ther Technol 3: 25, 2011.

17. Mankin HJ, Dorfman H,LippielloL and Zarins A: Biochemical and metabolic abnormalities in articular cartilage from osteo-arthritic human hips. II. Correlation of morphology with biochemical and metabolic data. J Bone Joint Surg Am 53: 523-537, 1971.

18. Livak KJ and Schmittgen TD: Analysis of relative gene expression data using real-time quantitative PCR and the 2(-Delta Delta C(T)) method. Methods 25: 402-408, 2001.

19. Longo UG, Loppini M, Fumo C, Rizzello G, Khan WS Maffulli $\mathrm{N}$ and Denaro V: Osteoarthritis: New insights in animal models. Open Orthop J 6: 558-563, 2012.

20. Orth P, Cucchiarini M, Zurakowski D, Menger MD, Kohn DM and Madry H: Parathyroid hormone [1-34] improves articular cartilage surface architecture and integration and subchondral bone reconstitution in osteochondral defects in vivo. Osteoarthritis Cartilage 21: 614-624, 2013.

21. Roudier M, Li X, Niu QT, Pacheco E, Pretorius JK, Graham K, Yoon BR, Gong J, Warmington $\mathrm{K}$, Ke HZ, et al: Sclerostin is expressed in articular cartilage but loss or inhibition does not affect cartilage remodeling during aging or following mechanical injury. Arthritis Rheum 65: 721-731, 2013.

22. van Bezooijen RL, Roelen BA, Visser A, van der Wee-Pals L, de Wilt E, Karperien M, Hamersma H, Papapoulos SE, ten Dijke P and Löwik CW: Sclerostin is an osteocyte-expressed negative regulator of bone formation, but not a classical BMP antagonist. J Exp Med 199: 805-814, 2004.

23. Li X, Zhang Y, Kang H, Liu W, Liu P, Zhang J, Harris SE and Wu D: Sclerostin binds to LRP5/6 and antagonizes canonical Wnt signaling. J Biol Chem 280: 19883-19887, 2005. 
24. Li X, Ominsky MS, Warmington KS, Morony S, Gong J, Cao J, Gao Y, Shalhoub V, Tipton B, Haldankar R, et al: Sclerostin antibody treatment increases bone formation, bone mass, and bone strength in a rat model of postmenopausal osteoporosis. J Bone Miner Res 24: 578-588, 2009.

25. Bouaziz W, Funck-Brentano T, Lin H, Marty C, Ea HK, Hay E and Cohen-Solal M: Loss of sclerostin promotes osteoarthritis in mice via beta-catenin-dependent and -independent Wnt pathways. Arthritis Res Ther 17: 24, 2015.

26. Chan BY, Fuller ES, Russell AK, Smith SM, Smith MM Jackson MT, Cake MA, Read RA, Bateman JF, Sambrook PN and Little CB: Increased chondrocyte sclerostin may protect against cartilage degradation in osteoarthritis. Osteoarthritis Cartilage 19: 874-885, 2011.

27. Mao B, Wu W, Davidson G, Marhold J, Li M, Mechler BM, Delius H, Hoppe D, Stannek P, Walter C, et al: Kremen proteins are Dickkopf receptors that regulate Wnt/beta-catenin signalling. Nature 417: 664-667, 2002.

28. Niehrs C: Function and biological roles of the Dickkopf family of Wnt modulators. Oncogene 25: 7469-7481, 2006.

29. Honsawek S, Tanavalee A, Yuktanandana P, Ngarmukos S, Saetan N and Tantavisut S: Dickkopf-1 (Dkk-1) in plasma and synovial fluid is inversely correlated with radiographic severity of knee osteoarthritis patients. BMC Musculoskelet Disord 11: $257,2010$.

30. Oh H, Chun $\mathrm{CH}$ and Chun JS: Dkk-1 expression in chondrocytes inhibits experimental osteoarthritic cartilage destruction in mice. Arthritis Rheum 64: 2568-2578, 2012.

31. Weng LH, Wang CJ, Ko JY, Sun YC and Wang FS: Control of Dkk-1 ameliorates chondrocyte apoptosis, cartilage destruction, and subchondral bone deterioration in osteoarthritic knees. Arthritis Rheum 62: 1393-1402, 2010.

32. Guo J, Liu M, Yang D, Bouxsein ML, Saito H, Galvin RJ, Kuhstoss SA, Thomas CC, Schipani E, Baron R, et al: Suppression of Wnt signaling by Dkk1 attenuates PTH-mediated stromal cell response and new bone formation. Cell Metab 11: 161-171, 2010.

33. Hwang SG, Ryu JH, Kim IC, Jho EH, Jung HC, Kim K, Kim SJ and Chun JS: Wnt-7a causes loss of differentiated phenotype and inhibits apoptosis of articular chondrocytes via different mechanisms. J Biol Chem 279: 26597-26604, 2004.
34. Voronkov A and Krauss S: Wnt/beta-catenin signaling and small molecule inhibitors. Curr Pharm Des 19: 634-664, 2013.

35. Zhang M, Xie R, Hou W, Wang B, Shen R, Wang X, Wang Q, Zhu T, Jonason JH and Chen D: PTHrP prevents chondrocyte premature hypertrophy by inducing cyclin-D1-dependent Runx2 and Runx3 phosphorylation, ubiquitylation and proteasomal degradation. J Cell Sci 122: 1382-1389, 2009.

36. Wan M, Yang C, Li J, Wu X, Yuan H, Ma H, He X, Nie S, Chang C and $\mathrm{Cao} \mathrm{X}$ : Parathyroid hormone signaling through low-density lipoprotein-related protein 6. Genes Dev 22: 2968-2979, 2008.

37. Tobimatsu T, Kaji H, Sowa H, Naito J, Canaff L, Hendy GN, Sugimoto $\mathrm{T}$ and Chihara K: Parathyroid hormone increases beta-catenin levels through Smad3 in mouse osteoblastic cells. Endocrinology 147: 2583-2590, 2006.

38. Romero G, Sneddon WB, Yang Y, Wheeler D, Blair HC and Friedman PA: Parathyroid hormone receptor directly interacts with dishevelled to regulate beta-Catenin signaling and osteoclastogenesis. J Biol Chem 285: 14756-14763, 2010.

39. Kim IS, Otto F, Zabel B and Mundlos S: Regulation of chondrocyte differentiation by Cbfa1. Mech Dev 80: 159-170, 1999.

40. Takeda S, Bonnamy JP, Owen MJ, Ducy P and Karsenty G Continuous expression of Cbfa1 in nonhypertrophic chondrocytes uncovers its ability to induce hypertrophic chondrocyte differentiation and partially rescues Cbfa1-deficient mice. Genes Dev 15: 467-481, 2001.

41. Kamekura S, Kawasaki Y, Hoshi K, Shimoaka T, Chikuda H, Maruyama Z, Komori T, Sato S, Takeda S, Karsenty G, et al: Contribution of runt-related transcription factor 2 to the pathogenesis of osteoarthritis in mice after induction of knee joint instability. Arthritis Rheum 54: 2462-2470, 2006.

42. Ueta C, Iwamoto M, Kanatani N, Yoshida C, Liu Y, Enomoto-Iwamoto M, Ohmori T, Enomoto H, Nakata K, Takada K, et al: Skeletal malformations caused by overexpression of Cbfa1 or its dominant negative form in chondrocytes. J Cell Biol 153: 87-100, 2001.

43. Li TF, Dong Y, Ionescu AM, Rosier RN, Zuscik MJ, Schwarz EM, O'Keefe RJ and Drissi H: Parathyroid hormone-related peptide (PTHrP) inhibits Runx2 expression through the PKA signaling pathway. Exp Cell Res 299: 128-136, 2004. 